\title{
NOT A S
}

\section{SOBRE LES REGLES D'ESQUIVAR VOCABLES I «LA QÜESTIÓ DE LA LLENGUA»*}

\author{
Julia Butiñá, Mercè Montagut y M. ${ }^{a}$ LlứSA ORDÓÑEZ
}

Si siempre es laborioso y delicado reseñar una obra de un maestro, lo es más aún en un caso como éste, en que a lo intrincado de la historia secular y crítica de la obra de que trata el Dr. Badia podría añadirse la que ha despertado ya este mismo estudio suyo en tan corto espacio de tiempo'. Nos anima, sin embargo, el hecho de darlo a conocer en el ámbito de la Filología Española, especialmente cuando esta voluntad de difusión se hace manifiesta en su autor desde el comienzo. Poner en relación este objeto de estudio con los estudios hispánicos es coherente con la línea del Dr. Badia como hispanista, aunque el interés del trabajo al que nos referimos alcanza al mundo de la romanística, concepto al que apelaba él mismo recientemente -en otra publicación del Institut d'Estudis Catalans- - al dar nueva vida a la revista Estudis Romànics ${ }^{2}$.

El título del libro recoge dos elementos unidos por la ley de causa-efecto: las Reglas que aquí se estudian y editan —una especie de Appendix Probi, fruto de la preocupación purista del siglo $\mathrm{XV}^{3}$-, que han sido conservadas en un

- Antoni M. Badia i Margarit, Institut d'Estudis Catalans, «Biblioteca Filològica», XXXVIII, Barcelona, 1999.

' Valga como muestra que el Dr. Badia se ha avanzado ya a inaugurar el diálogo, pues empieza a responder titulando su artículo de primicia: Entom de Pere Miquel Carbonell. Primer comentari sobre les «Regles d'esquivar vocables» (edició i estudi de 1999), en Miscel.tània Giuseppe Tavani 2, Estudis de Llengua i Literatura Catalanes, XLII, 2001, págs. 83-96. Diálogo que prevé largo -al igual que lo fue en su etapa inicial, 50 años atrás, como veremos-; lo cual reseñamos ahora cumpliendo aquella premonición, $y$, si bien no aspiramos a reflejarlo en su totalidad, sí empezamos destacando este carácter de sanísima querelle científica que va adquiriendo esta temática.

2 «'Romània', 'Romanitas', 'Romanística'», Estudis Romànics, XXI, 2000, págs. 7-22, pubłicación interrumpida desde 1987.

${ }^{3}$ Con esta comparación inicia Germà Colón —qujen también confía en despertar el interés de los romanistas-- el estudio alrededor del mismo tema, que dedica al Dr. Badia: «Les Regles d'esquivar vocables, Autoria $i$ entorn lingǘstic , Treballs de la Societat Catalana de Llengua $i$ Literatura, Barcelona, 2001, págs. 9-10.

RFE, LXXXIII, 2003, 3."-4. ${ }^{\circ}$, págs. 281-290 
códice formado por un conjunto heterogéneo de escritos de Pere Miquel Carbonell, que integran el manuscrito 69 del Archivo Capitular de la Catedral de Gerona, el cual incluye manuscritos pertenecientes a los años 1473-1507; y, por otro lado y tema muy principal, los aspectos relativos a la génesis de este tipo de normativas, que delatan las discusiones de los hombres cultos de aquella época sobre la cuestión de la lengua -tema que excede en mucho a las mismas reglas-.

Hay que empezar valorando en primer lugar algunos aspectos de esta exhaustiva investigación, los cuales resumiría en un par de notas muy concretas: transparencia y exigencia, amén de lo pulcrísimo de la edición entera, algo modélico en la época de las prisas. El curso de la misma lo ha definido acertadamente Eulàlia Duran al compararlo con el de una novela policíaca ${ }^{4}$, ya que el Dr. Badia la ha dotado de un ritmo que, unido al tono personal predominante - el autor coge al lector de la mano, lo guía y acompaña, explicándole sus íntimas motivaciones-, convierten al libro en una obra si no de intriga, sí de acendrado interés. Si los dos rasgos apuntados al principio asientan este libro como modélico en honestidad investigadora, este otro rasgo es valioso por mostrar una envidiable juventud y energía. Por otro lado, se utiliza un lenguaje llano, pues según se advierte desde el principio ( $\$ 7$, pág.19), dejando de lado las especialidades, se pretende ponerlo al alcance de muchos, a riesgo de ser tildado de elemental por los especialistas; el motivo es que afecta a varias ramas del saber filológico, e incluso a aspectos de cultura general.

En la Introducción expone el Dr. Badia el recorrido investigador alrededor de las Reglas, que inició hace unos 50 años cuando tuvo la iniciativa de editarlas ${ }^{5}$, edición a la que siguió un estudio lingüístico; anteriormente sólo se contaba con la noticia escueta de Jaume Massó i Torrents (1932). En la actualidad, tras diversos trabajos — suyos y de la crítica ( $\$ 7$, págs. 20-21)—, llega a articular con coherencia un libro de 500 páginas, en el que se decanta con mayor convicción en sus apreciaciones y arguye decididamente una hipótesis de autoría, según la cual nos propone, con profusión de argumentos, al archivero real e historiador barcelonés Pere Miquel Carbonell ${ }^{6}$ (1434-1517), quien además es el autor de la copia conservada. Este supuesto conlleva cuestionar la entidad de un debate, que se llegó a hacer legendario a lo largo de los siglos, en torno al cual gira la Primera Parte del estudio del Dr. Badia (Part Primera, págs. 25-88).

\footnotetext{
${ }^{4}$ Arxiu de Textos Catalans Antics, 19, 2000, págs. 715-719.

s Se publicaron en el Boletín de la Real Academia de Buenas Letras de Barcelona, ХII, 1950 , pags. 137-152.

- Autor de las Chròniques d'Espanya, elaboradas en colaboración con su primo, Jeroni Pau; se editaron póstumas, en 1547; hay edición crítica a cargo de Agustí Alcoberro, en la colección «Els Nostres Clàssics» (Barcelona, ed. Barcino, 1997).
} 
Aun tratando de resumir al máximo hay que referirse a los protagonistas de tan singular debate (Dramatis personae, § 1.5, págs. 33-37): además de Carbonell, el canónigo Jeroni Pau - hijo del poeta Andreu Gassull, secretario de Alfonso el Magnánimo--, adscrito a la cancillería real y a la curia pontificia, bien relacionado con Paolo Pompilio y otros humanistas italianos. También figura el poeta valenciano Bernat Fenollar - paladín de la lengua correcta en tierras valencianas-, quien habría escrito una acusación normativa ${ }^{7}$ contra el uso de palabras malsonantes, la cual tradicionalmente se había relacionado con la primera parte de nuestras Reglas; ello se debía a que el caballero Jaume Gassull -el cuarto personaje, adscrito al cenáculo del anterior- había dejado constancia de ello en su obra Lo procés de les olives.

La brama dels llauradors de l'Horta de València ${ }^{8}$, texto donde este último defiende el habla de la Huerta, era la respuesta con que se oponía a ciertas imposiciones linguísticas. Según hemos apuntado, éstas se atribuían a Fenollar a causa de una hipotética * Sentència, que el mismo Dr. Badia había identificado con nuestras Reglas. Ahora bien, el cotejo de los vocablos condenados con los que él mismo utiliza, así como hechos tales como que el poeta, por ingenioso que fuera, no era en rigor un humanista - como parece que se puede pensar que lo fuera el autor de aquéllas-, hacen ahora al Dr. Badia separar de modo contundente aquella sentencia de las reglas y, además, afirmarse en la autoría de Carbonell. Aparte de los ocho principales argumentos (págs. 135-148), son de mucho peso algunos detalles; aunque no sean definitivos, destacamos un par de ellos: uno, significativo por lo contundente, como es que una vez hable en primera persona («a mi, Pere Miquel Carbonell», pág. 111), y otro, elocuente por lo llano, pues en un total de veinte antroponímicos que forman el listado de formas correctas relativas a nombres personales se incluyen cuatro que le eran muy próximos: los de sus dos esposas, el de su amigo Jeroni y el suyo propio.

El Dr. Badia aclara asimismo el proceso de confección de las Reglas, en las que se captá bien la adaptación al catalán del método usado para el latín: criterio etimológico, condena de coloquialismos y vulgarismos, elegancia y unidad lingüísticas.

Para llegar a alguna de las conclusiones apuntadas, la Segunda Parte del estudio se ha dedicado al códice en el que se encuentran las Reglas y al «efecto de la cuestión de la lengua» (El còdex. El manuscrit de les Regles. La data, págs. 92-102).

\footnotetext{
'Es denominada Bandeig - 'rechazo' - por Martín de Riquer, quien dedica a esta literatura de debates un capitulo de su Historia de la Literatura Catalana (1964, III: Bemat Fenollar i els seus amics, págs. 321-364).

\& El texto se reproduce aquí (págs. 51-54), por lo que cualquier interesado en adentrarse en el tema - que repercute más allá de la historia de la lengua y de la cultura en el ámbito catalán- cuenta aquí con todos los elementos pertinentes.
} 
Las novedades que presenta esta parte del estudio son: la cuestión de la fecha de composición de las Reglas y la tesis de la autoría única.

Tras ponernos en antecedentes sobre los anteriores estudiosos del códice y sobre el hecho de que entre tanto escrito diverso ${ }^{9}$, el manuscrito de las Reglas -quizá por su brevedad-, pasara inadvertido a personajes de la talla de un Jaime de Villanueva (1807) o un Manuel de Bofarull (1875), el Dr. Badia entra de lleno en la cuestión de la datación textual de las mismas que, en su momento, Jaume Massó y M. Antònia Adroher propusieron que comprendiera el período situado entre 1473 y 1507 , lapso temporal que considera excesivo y que sugiere disminuir hasta situarlo entre 1473 y 1492 , basándose en diversas razones que expondrá detalladamente.

Para las Reglas, sería 1492 tal como se lo sugieren las fechas de los textos más cercanos a las mismas: las treinta páginas que rodean a las Reglas se mueven entre 1481-1492. Item más, cuatro hechos concomitantes le reafirman en su convicción sobre la fecha de 1492: el codicológico, ya que la mayoría de documentos está relacionada con esta fecha; el filológico, puesto que uno de los textos cercanos a las Regles es el Llunari de Bernat de Granollachs, que con toda probabilidad fue transcrito hacia 1490-91; el histórico, proporcionado por una anotación que alude a la rendición de Granada diez folios más allá de las Reglas; y el biográfico, sustentado en el hecho verificado de que Jeroni Pau -uno de sus autores- regresó a Barcelona en 1492.

Nos hace saber que las Reglas - cuyo modelo no es el Appendix Probi, desconocido en la época, sino la copia de una lista alfabética de errores de escritura en latín seguidos de su forma correcta que está también recogida en el mismo códice - están contenidas en tres folios, escritos a triple columna y que presentan el vocablo erróneo seguido de su correcta forma: canyèm per cànem (regla 30, pág. 110), ça casa per la casa (166, pág. 119), no mantienen relación temática alguna con los textos que las preceden o las siguen, y presentan una uniformidad de autoría caligráfica y de estilo, características que se repetirán en las dos partes del listado, cuya muy cuidada reproducción facsímil es de gran ayuda para situar al estudioso en un texto que ha sido transcrito topográficamente (Edició facsímil de les Regles, pág. 103).

El impecable estudio filológico llevado a cabo por el Dr. Badia no pierde de vista la cuestión tan novedosa que ha propuesto como es la de la autoría única: la unidad del texto que proporcionan la coherencia textual (terminológica y ortográfica) e ideológica (respecto al empleo de la lengua correcta que llevará a desarrollar el catalán como «buena lengua», como lengua de cultura), que propicia la uniformidad de estilo, de criterio corrector de cada parte de

9 «libreta de apuntes, copiador de cartas, colección de datos de interés, inventario de exitos profesionales», como apunta Badia en $\S 7.1 .3$. 
las Reglas, es la clave que lleva al Dr. Badia a la tesis del autor único ( 87.4 , pág. 97, y $\$ 9.10-9.12$, págs. 148-154) en la persona de Pere Miquel Carbonell, en contra de la doble autoría propuesta hasta ese momento y apoyada por la presencia de los nombres de los dos autores y por presentar el repertorio dos partes diferenciadas por la palabra $\tau \varepsilon \lambda \hat{\circ}$.

El capítulo XI, con el que se inicia la Parte Tercera, es un estudio gramatical sobre las Reglas que, como dice Germà Colon ${ }^{10}$, es un pequeño resumen de gramática histórica catalana; estudia la fonética, la fonética histórica, la evolución del léxico y la morfosintaxis.

En fonética, por ejemplo, distingue los problemas de mera grafía como Pera, Pere, vaguer, veguer y otros muchos, de los de evolución histórica en palabras como ànimo (del latín animus-i); y de las evoluciones irregulares tales como fenoll / fonoll o metátesis como longaniça / langoniça.

Estudia fenómenos morfológicos: el artículo, las formas verbales y otras partes de la oración. Por ejemplo, habla de la distinción caber / cabre o la contraposición del pretérito simple y el perifrástico aní e venguí/vaig anar e vaig venir.

El capítulo XIII está dedicado al detallado estudio filológico, histórico-lingüístico y sociolingüístico de las Reglas, de cada una de las reglas, para averiguar en qué criterio se basaba el autor para aceptar o no una palabra. Los criterios aplicados en las 174 primeras reglas — atribuidas a Bernat Fenollar, idea que ahora rechaza el Dr. Badia - son algo diferentes a los de las de la segunda parte.

En la primera parte las correcciones están elaboradas según tres criterios diferentes: criterio gramatical, criterio ortografico y criterio fonético. En la segunda parte, como hemos apuntado anteriormente, cambian un poco ya que aparece también el criterio sociolinguíístico con frases como parlar de gent baixa, parlar de minyons, parlar de dona rústica.

Para Germà Colon las correcciones están hechas principalmente desde la óptica del valenciano, pero para Badia no está tan claro, idea que armoniza con su teoría de que el autor de las Reglas no es ni Fenollar ni J. Pau, sino Pere Miquel Carbonell.

Badia estudia las normas una por una y al final de cada comentario nos expone una conclusión en que explica cuál es el criterio seguido en cada norma.

Así, el primero es el de aceptar la forma más latinizante y condenar la más popular, de lo que tenemos un ejemplo en la regla 12-13 que acepta quasi y rechaza quaix que es la forma vulgar. El segundo, aceptar la forma culta en vez de la popular instrument en vez de strument. Tercer criterio, aceptar la for-

${ }^{10}$ Op. citt, pág. 15. 
ma corriente en vez de la forma demasiado culta o arcaica: regla núm. 38, acepta mirar forma corriente. frente a la forma arcaica guardar.

Defensa de la forma correcta en vez de un vicio de pronunciación, regla 23, hay que decir tapí en vez de petí, donde hay además una metátesis. Defensa de la forma culta por encima de la más general y usual, regla 54, no teich sino tysich.

Un caso peculiar se da en las normas 48 y 49 en donde rechaza el pretérito perifrástico vaig anar y acepta aní, forma esta última que aún se usa hoy en Valencia, mientras que en Cataluña se dice la primera. Esto demuestra que ya en el siglo XV se decía vaig anar, vas anar, etc.

También es curiosa la regla 174, donde introduce la palabra $\tau \varepsilon \lambda \circ \varsigma_{\text {, }}$ vocablo griego que significa 'fin', 'término', que parece que fue introducida por Carbonell; esto abonaría la tesis de Badia de que Carbonell es el autor intelectual de las Regles, ya que esta palabra aparece en todos sus escritos. A partir de aquí empiezan las normas atribuidas al canónigo y humanista Jeroni Pau.

En esta segunda parte los criterios parecen variar un poco, pues parece aceptar las formas generales en vez de las dialectales, por ejemplo en la regla 183 acepta lexar o dexar, forma general contra la forma jaquir, desechada como dialectal.

Más adelante, a partir de la norma 276, condena ciertas formas a las que les da el calificativo de parlar de minyons: de gom a gom, rinxo rinxo. En otras les pone el rótulo parlar de gent baixa o parlar de dona rústica, etc., con lo cual distingue distintos registros de la lengua.

El Dr. Badia en su estudio sobre el vocabulario atribuye un item determinado a cada término: si uno se caracteriza por preferir la forma culta sobre la forma vulgar, si otro prefiere la forma común a la dialectal y así con todos los términos. De tal modo que se puede establecer qué norma siguió el autor: Preferir la forma culta sobre la forma vulgar, la más frecuente. Preferir la forma latinizante, en vez de la forma corriente, casi tan frecuente como la anterior. A buena distancia, preferir la comín a la dialectal. Incluso, la forma común, a la demasiado arcaizante. Sostiene Badia que en muchas ocasiones las normas son sólo correcciones de defectos de pronunciación o de escritura.

En la segunda parte sigue la misma línea, pero encuentra otras nuevas que siguen un criterio diastrático: preferir la forma culta en vez de las formas rísticas o de gente baja o niños. O, diafásicamente, reyna, para la lengua normal, y regina para niveles de lengua culta, muy culta.

El ambiente que se hace explícito en todo este contexto humano, por encima de los hechos concretos relativos al episodio lingüístico en torno a las $R e-$ glas -al fin y al cabo, unas hojas de borrador sin sistematizar--, lleva al autor a cerrar el estudio con una última parte -la Cuarta-, en la que da relieve al hecho mismo que provocaba aquellas discusiones: «la questione della lin- 
gua» (págs. 397-458), en la que se encuadra la obra estudiada y que sintetiza el hálito humanístico de aquella época, bajo el cual —de acuerdo con la latinización de los espíritus "1-_, se rehabilitaba y depuraba el vulgar. A su vez, Antoni Ferrando ha estudiado la repercusión de aquel ambiente a través de las correcciones editoriales, tras la pista del decisivo papel de la imprenta para fijar la lengua ${ }^{12}$.

Cabe destacar las notas que se nos ofrecen acerca de los humanistas y el tratamiento de las lenguas vemáculas, en concreto sobre los humanistas de las tierras catalanas en el siglo XV, que se configuran aquí a la sombra del clímax napolitano de la corte del Magnánimo, alrededor del cual figuraban el $\mathrm{Pa}$ normita - preceptor suyo-, Lorenzo Valla, Leonardo Bruni d'Arezzo o Bartolomé Fazio ${ }^{13}$. Hay que valorar, pues, la aproximación que hace el Dr. Badia a estos círculos humanistas, sea del «eje» Valencia-Barcelona y sus relaciones con los italianos, que aparecen repetidamente; sea del círculo alfonsino de Nápoles o respecto a la curia pontificia, etc., especialmente por lo poco que han sido atendidos o estudiados ${ }^{14}$.

Dos de los principales aciertos del Dr. Badia, a nuestro entender, son aspectos que glosa y engarza con esta temática, aplicándolos al caso concreto, pero que ya había apuntado con anterioridad ${ }^{15}$; se trata del concepto de «humanista duro», que sólo emplea el latín (como era el caso de Jeroni Pau, pág. 13), con lo cual no queda excluido de la calificación humanista quien utiliza el romance, lengua a la que precisamente estaban algunos ya dignificando a la sombra del latín - cosa que, a fin de cuentas, se hizo general en etapas posteriores de normativización lingüística-; con el otro aspecto estoy aludiendo a la sintonía europea en cuanto a la sensibilidad hacia las lenguas romances.

$\mathrm{Si}$ el primero es importante porque contribuye a disolver el conflicto acerca de la existencia de un humanismo en vulgar ${ }^{16}$, el segundo lo es por insertar la

11 «La llatinització dels esperits» es el título del primer epígrafe de esta Cuarta Parte.

12 «El paper dels primers editors (1473-1523) en la fixació del català modern», Caplletra, 27: História de la Llengua, coord. por Miquel Nicolas, (1999, pags. 109-136); obviamente se refiere a menudo a las Reglas y también contrasta pareceres con este estudio del Dr. Badia.

${ }^{13}$ Con fragmentos de esta parte se encabeza la $2^{2}$ edición de la monografía de J. Butiñá, Tras los origenes del Humanismo: el *Curial e Glielfa», Madrid, UNED, 2000, pág. 14.

${ }^{14} \mathrm{Ya}$ lo valoró una estudiosa de estos entornos, Mariàngela Villalonga, en su primera intervención sobre esta obra, la cual concluía así: «El professor Badja reviu l'ambient de les lletres del Renaixement català de tal manera que fa sentir el lector partícip de les vivències dels nostres humanistes», «Parlar bé el catală al segle XV», Revista de Girona 203, 2000, pág. 84.

15 Así en «La impronta renacentista en las letras catalanas. Latín y romance en los siglos XV y XVI», Revista de Lenguas y Literaturas Catalana, Gallega y Vasca, 4, 1996, págs. 165-180.

${ }^{16}$ Cabe tener en cuenta que en el área filológica catalana ha habido en los últimos decenios un muy masivo revisionismo del concepto, en cuanto at humanismo catalán; si bien actualmente se considera que la tradición positiva (Rubió i Lluch, Rubió i Balaguer, Batlori, Riquer...) está 
producción hispánica en el conjunto occidental, dado que el Dr. Badia observa que -al margen de diferencias y calidades- en menos de cuatro decenios se escriben las principales defensas de cinco lenguas vulgares: Prose della volgar lingua (1525) de Pietro Bembo, Diálogo de la lengua (ca. 1535) de Juan de Valdés, el Diálogo em louvor da nossa linguagem (1540) de Joâo de Barros, Deffence et illustration de la langue françoyse (1549) de Joachim du Bellay y Los colloquis de la insigne ciutat de Tortosa (1557) de Cristòfor Despuig. Enfoque que brinda un último capítulo sobre L'afinat concert de les llengïes, en que se contrasta su sucesiva normalización.

Ahora bien, observemos que antes de la exultación de las lenguas, éstas habían dado ya monumentos literarios; baste citar el Decameron (1370) o Lo somni de Bernat Metge (1399). En la sensibilidad de aquellos hombres se daba lo que podríamos denominar también, más allá de la estrictamente lingüística, una "conciencia literaria» ${ }^{17}$. Como anticipan las primeras traducciones del Griselda-que Petrarca vuelve al latín desde el italiano boccaccesco y que Metge torna al romance dignificado, en catalán, desde el bello latín petrarquesco-, el hecho de la lengua se interrelaciona estrechamente con el literario y su uso o preferencia está cargado de significados, al margen del estricto traducir o verter a otra lengua; de modo que aquellos primeros humanistas no eran ajenos al hecho de que la realización textual, que tanto se preocupaban por ennoblecer, va íntimamente ligada a la elección de una u otra lengua ${ }^{18}$.

$\mathrm{Si}$-teniendo en cuenta la manifestación literaria, vinculada a la lingüística- - unimos esta etapa cuatrocentista, que se desarrolla en el estudio del Dr. Badia, con los peldaños previos - los que marcan las relaciones de la Cancillería con los estudios de Bolonia o bien a través de la curia papal de Aviñón, en la segunda mitad del siglo anterior-, aparecen prácticamente dibujados los períodos históricos que había esbozado Rubió i Balaguer y suscribió como historiador Miquel Batllori ${ }^{19}$. Conjunto bien delineado y de fuerte personalidad el del humanismo catalán, cuyo estudio es de esperar que, desde este impulso en

\footnotetext{
ya en buena parte recuperada, como expone Albert Hauf en La saviesa de Barllori, Valencia, ed. $\mathrm{Sac}, 2001$, pág. 52 .

17 No nos consta que se haya estudiado este matiz literario, mientras que la conciencia linguística se ha escudriñado ya desde diferentes ángulos (Lore Torrecini, Emma Martinell...). Por otro lado, las conciencias histórica y moral aparecen a menudo en ensayos sobre nuestras culturas, en contraposición a lo superficial o lo utilitarista que progresivamente impera; así, figuran a menudo en el precioso Di fronte ai classici, de Ivano Dionigi, Milán, 2002.

${ }^{18}$ Se aproxima al estudio de este enfoque J. Butiñá en Del Griselda catala al castellà, «Series Minors 7, Real Academia de Buenas Letras de Barcelona, 2002. (Entre esos primeros Griseldas cabría recordar la versión francesa de Philippe de Mézières, que se incluye -al igual que la catalana- en los quince años posteriores a la primera traducción, la petrarguesca).

19 Obra completa, vol. V, De l'Humanisme i del Renaixement, Valencia, ed. Tres i Quatre, 1995 , págs. $45-46$.
} 
los epígonos, vaya ganando en profundidad, se relacione con el panorama hispánico y se sistematice dentro del concierto europeo.

Los últimos puntos de nuestro libro son un resumen espléndido de Historia de la Lengua, Dialectología y Sociolinguística, que explican -insistimos: de modo magistral- - la evolución de la lengua nueva y el proceso literario ( $E l$ poder i la llengua, La fragmentació dialectal, El fermént castellà), todo ello muy oportunamente ubicado en relación con el tema estudiado. Estas escasas ocho páginas esclarecen los avatares de la lengua catalana én este final de la Edad Media, desde el que se determina en gran parte su devenir hasta nuestros días.

Ya acabando, desde el resquicio del colofón, voy a ejercer el papel de la subjetividad, en la medida que le sea permitido al autor de reseñas; primero, no para homologarme al Dr. Badia, pues fue mi maestro, sino para poner a su lado mi defensa de la autoría de otro humanista, embajador y caballero del Magnánimo - mossèn Gras-, como autor de la novela Curial e Güelfa ${ }^{20}$, y, a raíz de ello, manifestarle mi mayor no ya simpatía sino empatía, pues sé bien la seguridad y satisfacción que proporciona el convencimiento. En segundo lugar -como filóloga decantada preferentemente hacia el estudio literario-, para relevar la importancia de la polémica en la que nos inserta su estudio. Porque si en lo lingüístico tuvo el recorrido y los límites que aquí se señalan y desbrozan, pudo alcanzar muy serias derivaciones en el campo literario, lo que según mi parecer está también muy pendiente de estudiar. Observemos que en la Brama y su rechazo podríamos llegar a ver reflejadas una preciosa síntesis del mismo Tirant lo Blanch, obra que ennoblece las distintas formulas y registros de lengua y que, bajo el mejor sello clasicista, rompe una lanza a favor del vernáculo, llegando al punto de tomar las leyendas ovidianas a través de la versión catalana de un contemporáneo, de Joan Roís de Corella ${ }^{21}$. Aquellas discusiones y otras cosas por el estilo justifican que haya monumentos literarios, de otro modo difícilmente explicables, en lenguas aún pendientes de configuración normativa.

Y por último, entendiendo que el Dr. Badia deja abierto el tema incluso a las pequeñas sugerencias y contribuciones -actitud la de mover al diálogo crítico que he comenzado resaltando-, podría apuntar que he realizado un ejercicio muy fácil, como es contrastar las Reglas con el glosario que Ramon Aramon i Serra destacó del Curial e Güelfa, lo cual nos indica sólo dos frecuencias; en ellas, esta novela coincide con el criterio cultista (libera, Massella) del escrito de Carbonell, que era el propio de los humanistas. Este dato es

${ }^{20}$ Júlia Butinyà, «Sobre l'autoria del «Curial e Güielfa»", Boletín de la Real Academia de Buenas Letras de Barcelona, XLI, 1987-88, págs. 63-120.

${ }^{21}$ Su Prosa profana ha sido traducido recientemente al español por Vicent Martines, «Clásicos Medievales», Madrid, ed. Gredos, 2001. 
congruente con la tendencia a situar el autor lejos del sermo urbanus de BarceIona y Valencia, por lo que tras las características citadas no extrañaría vincularlo - una vez más por esta vía - a la corte humanista napolitana, a propósito de lo cual remito a la hipótesis recién citada sobre la autoría.

Ahora bien, estos comentarios son de preponderante cariz literario, mientras que la obra reseñada es preferentemente lingüística. Por ello, la parte principal de esta reseña - la referente a los capítulos centrales del libro (la Segunda y Tercera Parte del libro del Dr. Badia), que tratan estrictamente de las Reglas - ha corrido a cargo de Mercè Montagut y M. ${ }^{2}$ Lluìsa Ordóñez, que aprendieron también con el Dr. Badia y se han dedicado preferentemente a los estudios linguíisticos. 